\title{
Investigations of methyl lactate in the presence of reverse micelles by vibrational spectroscopy and circular dichroism ${ }^{\text {th }}$
}

\author{
Sergio Abbate ${ }^{\mathrm{a}, \mathrm{b}, *}$, Roberto Gangemi $^{\mathrm{a}, \mathrm{b}}$, France Lebon ${ }^{\mathrm{a}, \mathrm{b}}$, Giovanna Longhi ${ }^{\mathrm{a}, \mathrm{b}}$, Marco Passarello $^{\mathrm{a}, \mathrm{c}}$, \\ Angela Ruggirello ${ }^{c}$, Vincenzo Turco Liveri ${ }^{c}$ \\ a Dipartimento di Scienze Biomediche e Biotecnologie, Università degli Studi di Brescia, Viale Europa 11, 25123 Brescia, Italy \\ b CNISM, Consorzio Interuniversitario Scienze Fisiche della Materia, Via della Vasca Navale 84, 00146 Roma, Italy \\ “ Dipartimento di Chimica “S. Cannizzaro”, Università degli Studi di Palermo, Viale delle Scienze Parco d'Orleans II, 90128 Palermo, Italy
}

\section{A R T I C L E I N F O}

\section{Article history:}

Received 5 October 2011

Received in revised form 25 January 2012

Accepted 26 January 2012

Available online 4 February 2012

\section{Keywords:}

Methyl lactate

Sodium bis(2-ethylhexyl)sulfosuccinate

(AOT)

Reverse micelles

VCD

NIR

Anharmonicity

\begin{abstract}
A B S T R A C T
The FT-IR and vibrational circular dichroism (VCD) spectra of $(S)$ - and - $(R)$-methyl lactate have been recorded for neat samples and at various concentrations in $\mathrm{CCl}_{4}$ and DMSO solutions. These spectra are used to analyse the FT-IR and VCD spectra of methyl lactates in presence of sodium bis(2ethylhexyl)sulfosuccinate (AOT) in $\mathrm{CCl}_{4}$, where the surfactant molecules are known to form reverse micelles. Some tendency of methyl lactate to interact with AOT micellar aggregates is observed, but not as well defined as previously observed for dimethyl tartrate in analogous circumstances. Besides, near infrared (NIR) absorption and VCD data have been obtained for most of the above systems, allowing to monitor the first overtone of the $\mathrm{OH}$-stretching vibration of methyl lactates, especially in the spectroscopic region where the $\mathrm{OH}$ bond is not involved in hydrogen bond with other molecules. The NIR absorption and NIR VCD data for dilute solutions are reproduced and interpreted by anharmonic DFT calculations. Further understanding of the behavior of aggregated methyl lactate systems has been gained on this basis.
\end{abstract}

(c) 2012 Elsevier B.V. All rights reserved.

\section{Introduction}

$(S)$ - and (R)-methyl lactate (more often referred to as (L) and (D)-methyl lactate, respectively) have long been studied by vibrational circular dichroism (VCD) [1-5] and also recently Losada and $\mathrm{Xu}$ [6] have monitored by density functional theory (DFT) calculations the interaction of methyl lactate with few water molecules, justifying the VCD spectral changes in water solution with respect to the VCD spectra in $\mathrm{CCl}_{4}$ solution. Other techniques were also employed on these systems, in view of chiral recognition phenomena [7]. On the other hand a system supposedly similar to methyl lactate, dimethyl-tartrate, has recently been studied $[8,9]$ by FT-IR, mid-IR VCD and NMR in presence of two types of surfactant compounds, namely sodium bis(2-ethylhexyl)sulfosuccinate (AOT) and soy-lecithin in apolar solvents. The performed experiments have

\footnotetext{
is Work presented in part at ICAVS-6 International Conference, Sonoma County, CA, June 2011.

* Corresponding author at: Dipartimento di Scienze Biomediche e Biotecnologie, Università degli Studi di Brescia, Viale Europa 11, 25123 Brescia, Italy. Tel.: +39 303717415; fax: +39 303717416 .

E-mail address: abbate@med.unibs.it (S. Abbate).
}

been found very useful to monitor the strong interaction of both dimethyl tartrate enantiomers with AOT and lecithin polar heads.

The surfactant molecules are known to form reverse micelles when they are dissolved in apolar solvents [10]. As a consequence of anisotropic surfactant/surfactant interactions, their peculiar globular structure consists in a hydrophilic core formed by the surfactant head groups surrounded by its alkyl chains pointing outwards. Solutions of reverse micelles in apolar solvents are specialized solubilization systems because they offer a multiplicity of sites for hydrophilic, amphiphilic and hydrophobic substances. In the case of dilute solutions of amphiphilic compounds, like dimethyl tartrate or methyl lactate, their behavior is generally described in terms of a portioning of solute monomers between reverse micelles and apolar medium. On the other hand, in absence of surfactant, amphiphilic molecules are able to self-assemble in apolar media forming a variety of oligomers whose equilibrium populations are concentration dependent.

Then, one may ask whether it is possible to evaluate the composition and structure of the molecular aggregates, when both the surfactant and the amphiphilic substance coexist at relatively high concentration. This study, which had been undertaken with some success for dimethyl tartrate $[8,9]$, could be of some interest to the biologists, since reverse micelles have been proposed as realistic models of bio-compartments such as biomembranes. The aim of 


\section{AOT}<smiles>CCCCC(CC)CC(CC)COC(=O)C(CCCC)C(=O)O[NH3+]</smiles><smiles>COC(=O)[C@H](C)O</smiles>

R-ML

Scheme 1. Molecular structure of Sodium bis(2-ethylhexyl)sulfosuccinate (AOT), $(R)-(+)$-methyl lactate (R-ML) and (S)-(-)-methyl lactate (S-ML).

the present investigation is then to unveil the nature and composition of the aggregates present in methyl lactate/AOT/ $\mathrm{CCl}_{4}$ solutions. The structural formulae for both enantiomers of methyl lactate and AOT are given in Scheme 1.

As stated above, FT-IR, VCD, and NIR have proven as suitable techniques to provide structural information on the environment of spatially separated moieties of both solubilizate and surfactant molecules and consequently on the structural arrangements of their molecular aggregates in apolar solutions. In particular, AOT spectra are characterized by the environment sensitive $\mathrm{C}=\mathrm{O}, \mathrm{SO}_{3}{ }^{-}$ fundamental stretchings, and methyl lactate ones by the $\mathrm{OH}$ (fundamental and overtone) and $\mathrm{C}=\mathrm{O}$ stretchings.

\section{Materials and methods}

$(S)-(-)$-Methyl lactate (S-ML) and (R)-(+)-methyl lactate (R-ML) (Fluka, optical purity $>99 \%$ ) were used as received. Sodium bis(2ethylhexyl) sulfosuccinate (AOT, Sigma, 99\%, racemic mixture) was dried under vacuum for several days before use. To remove residual traces of water, solutions of AOT in $\mathrm{CCl}_{4}$ (Riedel-de Haën, 99.8\%) were gently stirred for several days in the presence of activated type 4A molecular sieves (Fluka, beads of 4 Å pore size). Solutions at various solubilizate-to-surfactant molar ratios $(\mathbf{R})$ were prepared by adding the appropriate amounts of $\mathrm{AOT} / \mathrm{CCl}_{4}$ solutions to a weighed quantity of S-ML or R-ML.

FT-IR spectra were recorded in the wavelength range $900-4000 \mathrm{~cm}^{-1}$ by a Perkin-Elmer (Spectrum BX) spectrometer using a fixed-path cell equipped with $\mathrm{CaF}_{2}$ windows. All measurements were performed at $25^{\circ} \mathrm{C}$ with a spectral resolution of $0.5 \mathrm{~cm}^{-1}$.

The VCD spectra in the mid-IR range were taken on $0.2 \mathrm{M} \mathrm{CCl}_{4}$ and DMSO solutions and on R-ML/AOT/CCl $/ \mathrm{Cl}_{4}$ and S-ML/AOT/CCl solutions at $\mathbf{R}=1$ and $\mathbf{R}=2$, (AOT concentration $0.082 \mathrm{M}$ ) in $\mathrm{BaF}_{2}$ $0.1 \mathrm{~mm}$ pathlength cells using a Jasco FVS 4000 apparatus. The spectra resulted from 2000 scans or from averages over a series of 4 runs, each one of 2000 scans, with a resolution of $4 \mathrm{~cm}^{-1}$. In all cases the IR and VCD spectra of the solvent or surfactant was subtracted.

Near infrared (NIR) absorption and VCD spectra were taken on a home-built apparatus with samples contained in $0.5-5 \mathrm{~cm}$ pathlength quartz cells on $\mathrm{R}-\mathrm{ML} / \mathrm{AOT} / \mathrm{CCl}_{4}$ and $\mathrm{S}-\mathrm{ML} / \mathrm{AOT} / \mathrm{CCl}_{4}$ solutions

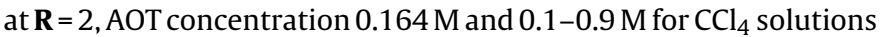
in the range $1600-1300 \mathrm{~nm}$ (first $\mathrm{OH}$-stretching overtone). Averages over 4 series, with the resolution of $2 \mathrm{~nm}, 2$ scans each were considered. Spectra were run by the procedures described in Refs. $[11,12]$ and for the micellar solution $(\mathbf{R}=2)$ the NIR absorption of the surfactant was subtracted.

DFT calculations for the prediction of the mid-IR VCD spectra for the isolated R-ML molecule were conducted with the B3LYP/6$311++G(d, p)$ level of theory in vacuo and with the IEF-PCM solvation model of Gaussian 09 package [13] in the harmonic approximation. No scaling factor was applied to calculated frequencies and calculated spectra were constructed by assigning Lorentzian bandshapes to predicted vibrational transitions with bandwidths of $10 \mathrm{~cm}^{-1}$. The anharmonicity calculations were run with our own codes and Gaussian 09, as described in Refs. [14,15], and allowed us to evaluate the mechanical anharmonicity parameter $\chi$ and various electrical anharmonic parameters: with these we were able to predict the first OH-stretching overtone NIR and NIR-VCD spectra.

\section{Results and discussion}

\subsection{IR data}

To evidence the concentration dependence of the $\mathrm{OH}$ stretching band in the fundamental $\mathrm{OH}$-stretching region, we have normalized the spectra in such a way that the absorbance maximum is in all cases $A=1$ : we show them in Fig. 1 for neat $S-M L$ and for $S-M L$ solutions in $\mathrm{CCl}_{4}$ at three concentrations (panel $\mathrm{A}$ ), and in solution of DMSO- $\mathrm{d}_{6}(0.2 \mathrm{M})$ and in $\mathrm{AOT} / \mathrm{CCl}_{4}$ solutions at fixed surfactant concentration $([\mathrm{AOT}]=0.164 \mathrm{M})$ (panel $\mathrm{B}$ ) (in the latter case we also repeat the neat liquid data for ease of comparison).

One may notice that the band shape is influenced by the type of solvent $\left(\mathrm{CCl}_{4} v s\right.$. DMSO- $\left.\mathrm{d}_{6}\right)$, by increasing the concentration of $\mathrm{S}-\mathrm{ML}$ in $\mathrm{CCl}_{4}$, as well as by the presence of AOT. In particular, the most marked spectral contribution of the lower wavenumber broad components ( $c a .3455 \mathrm{~cm}^{-1}$ and $3312 \mathrm{~cm}^{-1}$ ) occurring in AOT micellar solutions and in DMSO (ca. $\left.3305 \mathrm{~cm}^{-1}\right)$ and in the neat liquid state $\left(\mathrm{ca} .3470 \mathrm{~cm}^{-1}\right)$ are tentatively assigned to intermolecular hydrogen bonded $\mathrm{OH}$ stretchings. In particular the band at $c a .3470 \mathrm{~cm}^{-1}$, found in the neat liquid state and in the threecomponent solutions, is assigned to $\mathrm{OH}$ stretchings perturbed by intermolecular S-ML/S-ML dimer, trimer, etc. formations; the ca. $3305 \mathrm{~cm}^{-1}$ band found in DMSO solutions bears some similarity to the low wavenumber component of the structured band observed in the three-component solutions and thus is assigned to $\mathrm{OH}$ stretchings perturbed by intermolecular $\mathrm{S}-\mathrm{ML} / \mathrm{SO}_{3}{ }^{-}$associations (or by the only one $S=0$ in DMSO- $\mathrm{d}_{6}$ ). This means that in presence of AOT, S-ML competes in bonding to either other S-ML molecules or to the head groups of the surfactant AOT molecules. Next, one may notice for S-ML/CCl 4 solutions, as well as for S-ML in presence of the surfactant, the existence of a sharp absorption band at $c a .3550 \mathrm{~cm}^{-1}$ (marked $\alpha$ in Fig. 1), which will be shown later to be typical of the $\mathrm{OH}$-stretching of the single S-ML molecule forming $\mathrm{OH} \ldots \mathrm{O}=\mathrm{C}$ intramolecular hydrogen bond, as attributed before [16]. Another less intense band at ca. $3600 \mathrm{~cm}^{-1}$ (marked $\beta$ in Fig. 1), attributed to S-ML molecule forming $\mathrm{OH} \ldots \mathrm{OCH}_{3}$ intramolecular hydrogen bond, is also noticed here [16]. In conclusion, it may be assumed with some confidence that, in the presence of AOT reverse micelles, S-ML molecules dispersed in the bulk $\mathrm{CCl}_{4}$ solvent, either in isolation or associated with other S-ML molecules, coexist with those confined in the micellar aggregates. Other forms of spectroscopic experiments presented in this work will provide some more details on the two types of associations. Moreover, since the $\mathrm{O}-\mathrm{H}$ stretchings frequencies of confined S-ML molecules in reverse micelles are lower than the $\mathrm{O}-\mathrm{H}$ stretching frequencies of S-ML in the liquid state, it may be argued that methyl lactate hydroxyls experience stronger interactions in the micelle environments than in the liquid state. The frequencies of the observed bands are reported in Table 1 together with the first overtone data, which will be commented later. No significant spectral difference between the $\mathrm{OH}$ band of the two enantiomers has been detected implying that 

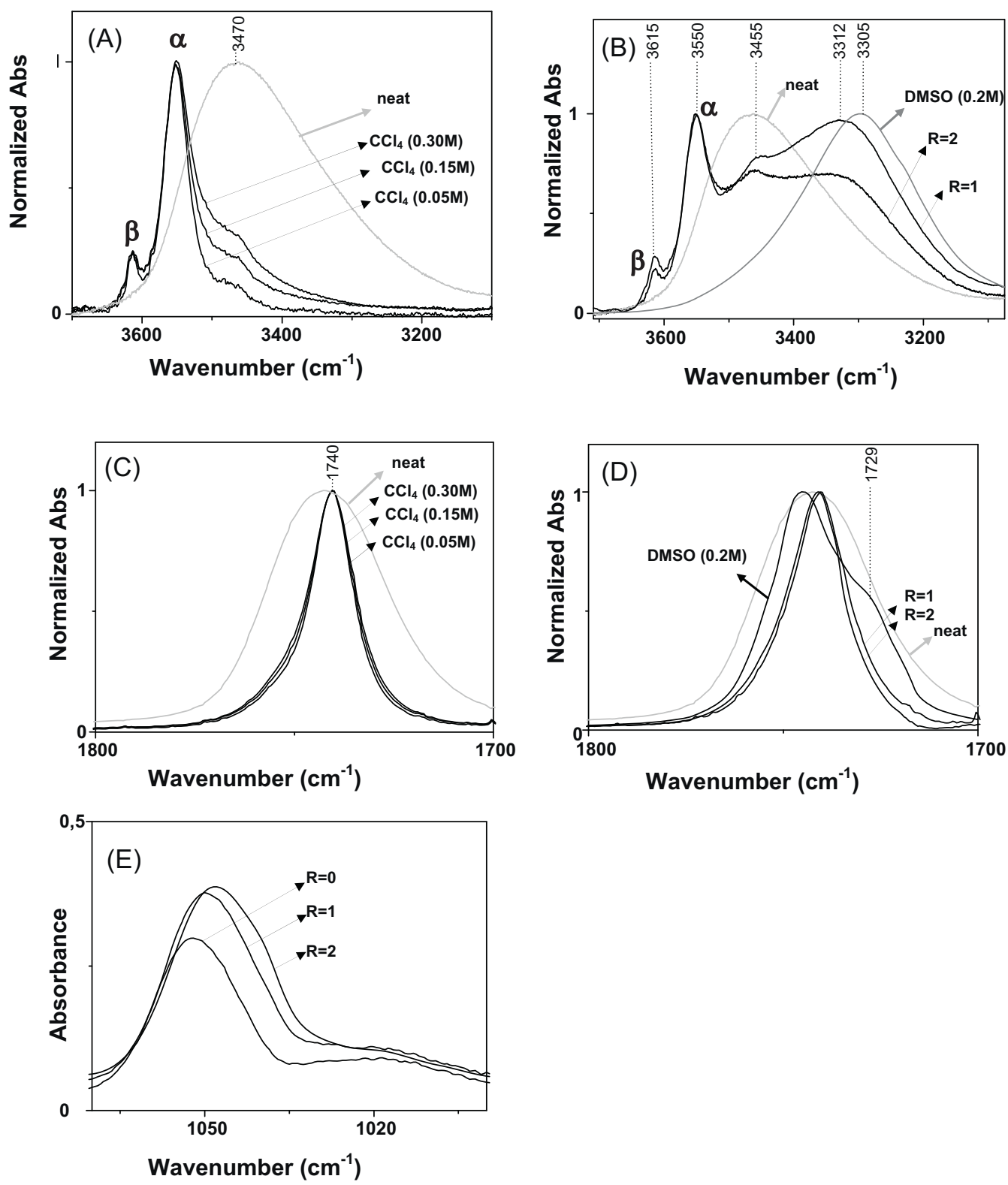

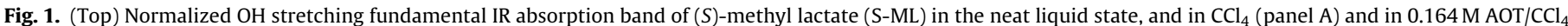

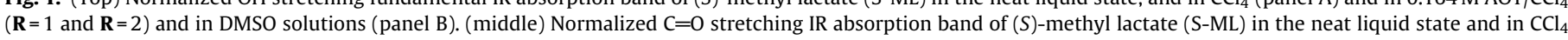

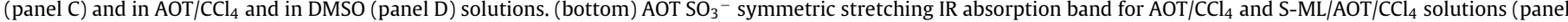
E).

the $\mathrm{OH}$ groups of both compounds experience the same local environment. (Commercial AOT is a diastereomeric mixture: no chiral recognition has been evidenced by our experiments.)

Whether and how the $\mathrm{C}=0$ group of $\mathrm{S}-\mathrm{ML}$ probes the immediate surroundings may be monitored by looking at its $C=0$ stretching band occurring at about $1740 \mathrm{~cm}^{-1}$. The $\mathrm{S}-\mathrm{ML} \mathrm{C}=\mathrm{O}$ stretching band has been obtained by subtracting the spectrum of $\mathrm{AOT} / \mathrm{CCl}_{4}$ solution from those of S-ML/AOT/CCl ${ }_{4}$ samples. These bands, normalized such that the maximum absorbance $A$ is 1 , are shown in Fig. 1, panels $C$ and $D$ together with that for the solution in DMSO- $\mathrm{d}_{6}$ and for neat liquid S-ML. With respect to the $\mathrm{CCl}_{4}$ solution, noticeable band broadening is observed, especially for neat S-ML, where also a small blue shift is observed to $1745 \mathrm{~cm}^{-1}$ (with an FWHH of $\left.c a .36 \mathrm{~cm}^{-1}\right)$ and an additional blue shift $\left(6 \mathrm{~cm}^{-1}\right)$ is observed for S-ML in DMSO- $\mathrm{d}_{6}$, with also a major shoulder growing in at $1729 \mathrm{~cm}^{-1}$ (as observed previously for dimethyl tartrate [9]). Some band broadening ( $\mathrm{ca} .15 \mathrm{~cm}^{-1} \mathrm{FWHH}$ ) is also observed in presence of AOT. These facts suggest that part of the S-ML molecules are in part inter-molecularly H-bonded to other S-ML molecules and in part are bonded to the polar heads of the surfactant molecule. This interpretation is consistent also with the data of the $\mathrm{OH}$-stretching region.

Finally, the band of the AOT $\mathrm{SO}_{3}{ }^{-}$symmetric stretching absorption in S-ML/AOT/ $\mathrm{CCl}_{4}$ samples exhibits a shift to lower frequencies with increasing S-ML concentration together with some intensity enhancement (see Fig. 1 panel E). The observed frequency behavior, similar to that observed when an increasing amount of water is solubilized within AOT reverse micelles [17], indicates that on the average there are more interacting S-ML molecules per AOT.

\subsection{VCD data in the mid-IR}

In Fig. 2 we report the comparison of IR and VCD spectra of R$\mathrm{ML}$ and S-ML in $\mathrm{CCl}_{4}$ (panel a) in the $1500-900 \mathrm{~cm}^{-1}$ range to the 

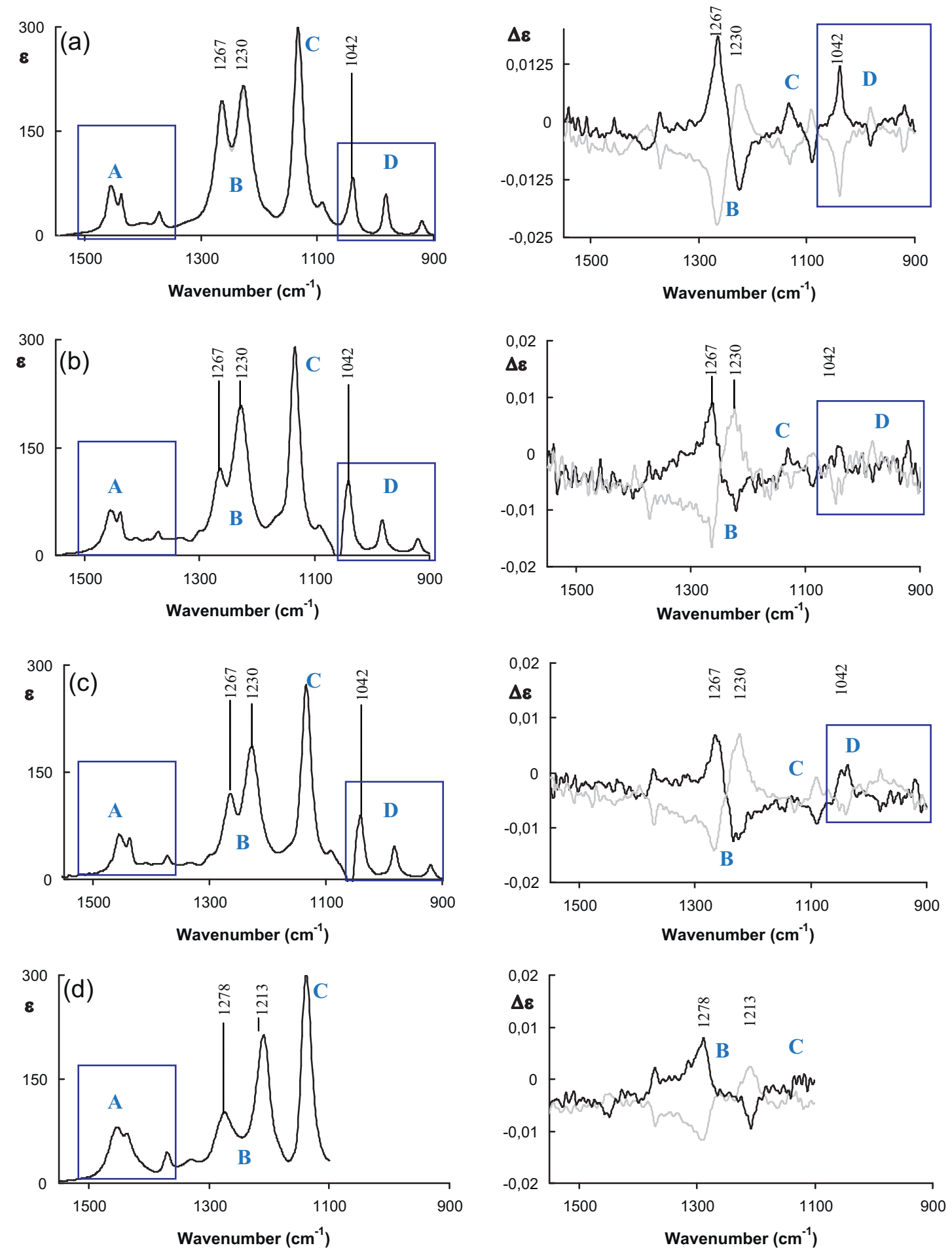

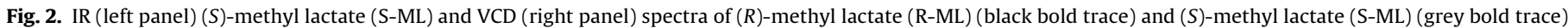
in (a) $0.2 \mathrm{M} / \mathrm{CCl}_{4}$ solution, (b) in $\mathbf{R}=1$ micelles, (c) in $\mathbf{R}=2$ micelles, and (d) in $0.2 \mathrm{M} / \mathrm{DMSO}$ solution.

spectra of $\mathbf{R}=1$ and $\mathbf{R}=2 \mathrm{ML} / \mathrm{AOT} / \mathrm{CCl}_{4}$ systems in the same range (panels b and c respectively), the AOT/CCl4 spectrum has been subtracted. For sake of discussion we also report the IR and VCD spectra of R-ML and S-ML in DMSO-d 6 (panel d).

DMSO by itself is not really a model for AOT polar heads: chemical groups in DMSO and AOT are different and the molar ratios ML/AOT and ML/DMSO are different as well. However in DMSO solutions we can study spectral signals of ML molecules in presence of a strong hydrogen acceptor; thus the comparison of spectra presented in Fig. 2 can be instructive (as in an analogous instance examined in Refs. $[8,9])$.

Some changes in the behavior of IR and VCD spectra of R-ML and $\mathrm{S}$-ML in presence of AOT are observed with respect to what happens in $\mathrm{CCl}_{4}$ solutions, but not as drastic as observed for dimethyl tartrate in the same conditions [8,9]; a more marked change is found for VCD spectra in DMSO- $\mathrm{d}_{6}$ solutions (in all cases the VCD spectra of 
Table 1

Wavelength $(\mathrm{nm})$ and frequencies $\left(\mathrm{cm}^{-1}\right)$ of observed $\mathrm{OH}$-stretching overtone absorption bands for $0.1 \mathrm{M}, 0.9 \mathrm{M}$ solutions, neat ML and ML in presence of surfactant: a comparison is also provided with predicted frequencies from observed fundamentals in the IR spectra shown in Fig. 1 and $\chi=88.6 \mathrm{~cm}^{-1}$, as explained in the text.

\begin{tabular}{|c|c|c|c|}
\hline $\begin{array}{l}v_{\mathrm{obs}}\left(\mathrm{cm}^{-1}\right) \\
\Delta v=1\end{array}$ & $\begin{array}{l}\lambda_{\text {obs }}(\mathrm{nm}) \\
\Delta v=2\end{array}$ & $\begin{array}{l}v_{\mathrm{obs}}\left(\mathrm{cm}^{-1}\right) \\
\Delta v=2\end{array}$ & $v_{\text {pred }}\left(\mathrm{cm}^{-1}\right) \Delta v=2$ \\
\hline \multicolumn{4}{|l|}{$\mathrm{CCl}_{4}(0.1 \mathrm{M})$} \\
\hline$v_{\gamma}$ & 1381 br. & 7236 & $7170(1394 \mathrm{~nm}) v_{\alpha}+v_{\beta}$ \\
\hline$v_{\beta}=3617$ & 1415 & 7067 & $7057(1417 \mathrm{~nm}) 2 v_{\beta}$ \\
\hline $\begin{array}{l}v_{\alpha}=3553 \\
v=3473\end{array}$ & 1442 & 6935 & $\begin{array}{l}6929(1443 \mathrm{~nm}) 2 \nu_{\alpha} \\
6769(1477 \mathrm{~nm}) 2 \nu\end{array}$ \\
\hline \multicolumn{4}{|l|}{$\mathrm{CCl}_{4}(0.9 \mathrm{M})$} \\
\hline & 1379 br. & 7252 & \\
\hline & 1418 & 7052 & \\
\hline & 1444 & 6925 & \\
\hline \multicolumn{4}{|l|}{ Neat } \\
\hline & 1374 br. & 7278 & \\
\hline & 1445 & 6020 & \\
\hline \multicolumn{4}{|l|}{ With AOT } \\
\hline$v_{\gamma}$ & 1377 br. & 7262 & $7162(1396 \mathrm{~nm})$ \\
\hline$v_{\beta}=3618$ & 1416 & 7062 & $\begin{array}{l}v_{\alpha}+v_{\beta} \\
7059(1417 \mathrm{~nm}) \\
2 v_{\beta}\end{array}$ \\
\hline$v_{\alpha}=3544$ & 1443 & 6930 & $\begin{array}{l}6911(1447 \mathrm{~nm}) \\
2 v_{\alpha}\end{array}$ \\
\hline
\end{tabular}

S-ML and R-ML are acceptable mirror images). First of all, the IR spectra in the four cases of Fig. 2 are quite similar, except for the region labelled $\mathrm{B}$, where the two bands observed at $\mathrm{ca}$. 1267 and $1230 \mathrm{~cm}^{-1}$ have almost equal intensities in $\mathrm{CCl}_{4}$ solutions, while they have rather different intensities in the other three cases: in DMSO- $\mathrm{d}_{6}$ the two bands are also more separated and the $1230 \mathrm{~cm}^{-1}$ component moves to $1213 \mathrm{~cm}^{-1}$, while the $1267 \mathrm{~cm}^{-1}$ moves to $1278 \mathrm{~cm}^{-1}$. The regions labelled A, C and D do not show significant changes in the IR spectra, except for the $1042 \mathrm{~cm}^{-1}$ band in the D region changing in intensity, as commented in the previous section, due to the perturbation by the $\mathrm{SO}_{3}{ }^{-}$stretchings of AOT underneath; in the DMSO solutions, $\mathrm{S}=\mathrm{O}$ stretchings are so strongly absorbing in region $\mathrm{D}$ that we can report neither IR nor VCD data there. The VCD spectra show parallel changes, in other words in region $B$ the relative VCD intensity of the 1267 to the $1230 \mathrm{~cm}^{-1}$ bands changes, even though in the opposite way with respect to the IR spectra. In DMSO- $\mathrm{d}_{6}$ solution the two VCD bands get also more separated in frequency and change to a larger extent than in presence of AOT. Finally, the VCD band in correspondence of the $1042 \mathrm{~cm}^{-1}$ IR band decreases in intensity: this change may be very well due to changes brought in by the $\mathrm{SO}_{3}{ }^{-}$moiety, that we monitored previously in the IR spectra. As it was concluded in first paragraph of this section, we may state that, while a strong interaction between the $S=0$ groups of DMSO and S-ML (or R-ML) molecules occurs, the same happens to a much lesser extent between AOT and S-ML (or R-ML), and this means to us that some S-ML molecules interact with AOT molecules and some do not.

In order to clarify the origin of the observed changes in the mid-IR we have performed some $a b$-initio calculations. Some time ago, HF/and DFT/harmonic approximations have been already reported for ML in vacuo [1-6] and for $\mathrm{ML}+\mathrm{H}_{2} \mathrm{O}$ complexes [6]. We have repeated the calculations for the isolated molecule and have obtained, as agreed in the literature [1-6], that the major conformer ( $c a .90 \%$ population) shows a kind of internal hydrogen bond between the $\mathrm{OH}$ and $\mathrm{C}=\mathrm{O}$ moieties, i.e. a short $\mathrm{OH} \cdots \mathrm{O}=\mathrm{C}$ distance. Two other conformers appear, with the $\mathrm{OH}$ bond approaching the oxygen atom of the $\mathrm{OCH}_{3}$ moiety (see Table 2), being slightly tilted on each of the two sides of the OCC plane. We verified by the $\mathrm{PCM}-\mathrm{IEF}$ approach both in $\mathrm{CCl}_{4}$ and in DMSO that essentially the same conformers are obtained, but with significantly different population factors. The calculations for the DMSO case indicate the presence of two new conformers (4 and 5) without intramolecular $\mathrm{H}$ bond but their contribution to the total population remains very low (4\%).

The experimental and calculated IR and VCD spectra in $\mathrm{CCl}_{4}$ and DMSO- $\mathrm{d}_{6}$ are presented in Fig. 3 (left and right panel, respectively) (see also Fig. S1 of Supporting Information). The predicted IR and VCD spectra for the $\mathrm{CCl}_{4}$ solvent is in good agreement with the experiment except the too high intensity for the IR signals at 1249 and $1274 \mathrm{~cm}^{-1}$. For the DMSO- $\mathrm{d}_{6}$ solvent, the predicted IR and VCD spectra fail to reproduce the experimental spectra between 1100 and $1300 \mathrm{~cm}^{-1}$.We know that modelling of the DMSO solvent should be made by explicitly placing DMSO molecules close to S$\mathrm{ML}$, as indicated by Polavarapu for this kind of systems $[3,18]$. We report our calculations here just for completeness. Finally and interestingly, the normal mode analysis of the results of DFT calculations allows to understand that the regions $\mathrm{B}$ and $\mathrm{D}$ (where changes in the IR and VCD spectra occur) always involve the $\mathrm{OH}$ bending.

\subsection{NIR absorption and NIR-VCD data}

In Fig. 4 (left panel) we superimpose the experimental NIR absorption (top) and NIR-VCD spectra (bottom) of S-ML and R-ML as neat liquids or as $0.1 \mathrm{M}$ and $0.9 \mathrm{M} \mathrm{CCl}_{4}$ solutions in the region 1300-1600 nm, corresponding to the first $\mathrm{OH}$-stretching overtone. For ease of comparison the data have been reported in $\varepsilon$ and $\Delta \varepsilon$ ordinate scales.

In Fig. 4 (right panel) we superimpose the NIR(top) and NIR-VCD (bottom) spectra of $\mathbf{R}=2 \mathrm{ML} / \mathrm{AOT} / \mathrm{CCl}_{4}$ systems. To the corresponding spectra of $0.9 \mathrm{M} / \mathrm{CL}_{4}$ solutions of S-ML and R-ML (the latter spectra being the most similar to those for the three-component system): from the absorption spectra of $\mathrm{ML} / \mathrm{AOT} / \mathrm{CCl}_{4}$ the absorption spectrum due to $\mathrm{CH}$ stretching-bending combinations has been subtracted. This region contains the $\mathrm{OH}$-stretching first overtone transition of the monomeric molecule and we assign the two main peaks at $c a .1410$ and $1440 \mathrm{~nm}$ to the three $\mathrm{OH}$-stretchings in the most populated conformational states (the $\mathrm{OH}$ stretching overtones for inter-molecularly bonded molecules are expected to appear in twice a broader region than in the IR fundamental region, and it is known [19] that they show a much lower intensity): the characteristic frequencies and absorption and VCD intensities are read comfortably from the spectra of the most diluted solutions (left panels) and will be quantitatively investigated below. More specifically we assign the most intense peak (that we call $\alpha$ ) both in the NIR and in the NIR-VCD spectra at $1440 \mathrm{~nm}$ to the $\mathrm{OH}$ stretching overtone for the $\mathrm{OH}$ intra-molecularly bonded to the $\mathrm{C}=\mathrm{O}$ (see Table 2). The other much less intense band (that we call $\beta$ ) observed at $c a .1410 \mathrm{~nm}$ is assigned to the $\mathrm{OH}$-stretching for the $\mathrm{OH}$ bond in the other two conformational states (see Table 2). The band at ca. $1377 \mathrm{~nm}$ (that we call $\gamma$ ) appearing in all solution cases as well as in the ternary systems may be due to a combination state. Indeed, as reported in Table 1, the two former features $\alpha$ and $\beta$ occur at frequencies close to two times those for the corresponding bands in the fundamental region in the IR spectrum minus twice the anharmonicity $\chi=88.6 \mathrm{~cm}^{-1}$ (the latter value was calculated for borneol recently $[14,15])$. Also in the neat liquid state there is some NIR-VCD signature of the isolated molecule; here and in the rest of the cases the sign of the VCD band does not change. Let us discuss the data of Table 1 more closely. Since we observe in Fig. $1 \mathrm{~A}$ and $\mathrm{B}$, for the monomeric molecule, the same pattern in the $\mathrm{OH}$-stretching fundamental region as in the first overtone $\mathrm{OH}$-stretching region, we make the same assignment in the fundamental region and reconstruct from Birge-Sponer law [20] the $\mathrm{OH}$-stretching overtone band centers as functions of the $\mathrm{OH}$-stretching fundamental band centers; namely if we call $v_{1}$ the frequency for the fundamental and $v_{2}$ 
Table 2

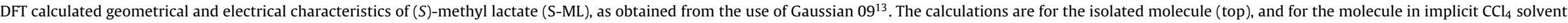
(middle) or DMSO solvent (bottom). For the definition of symbols see text.

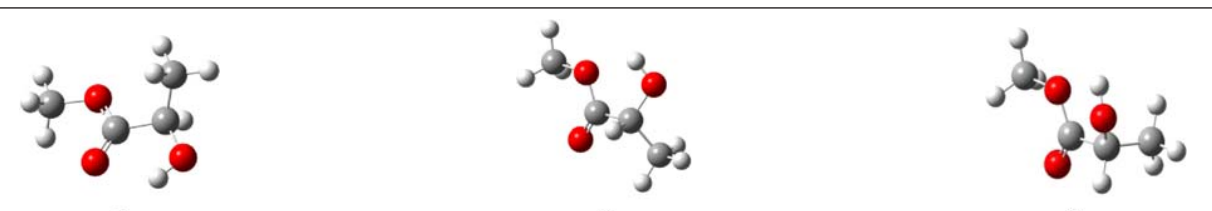

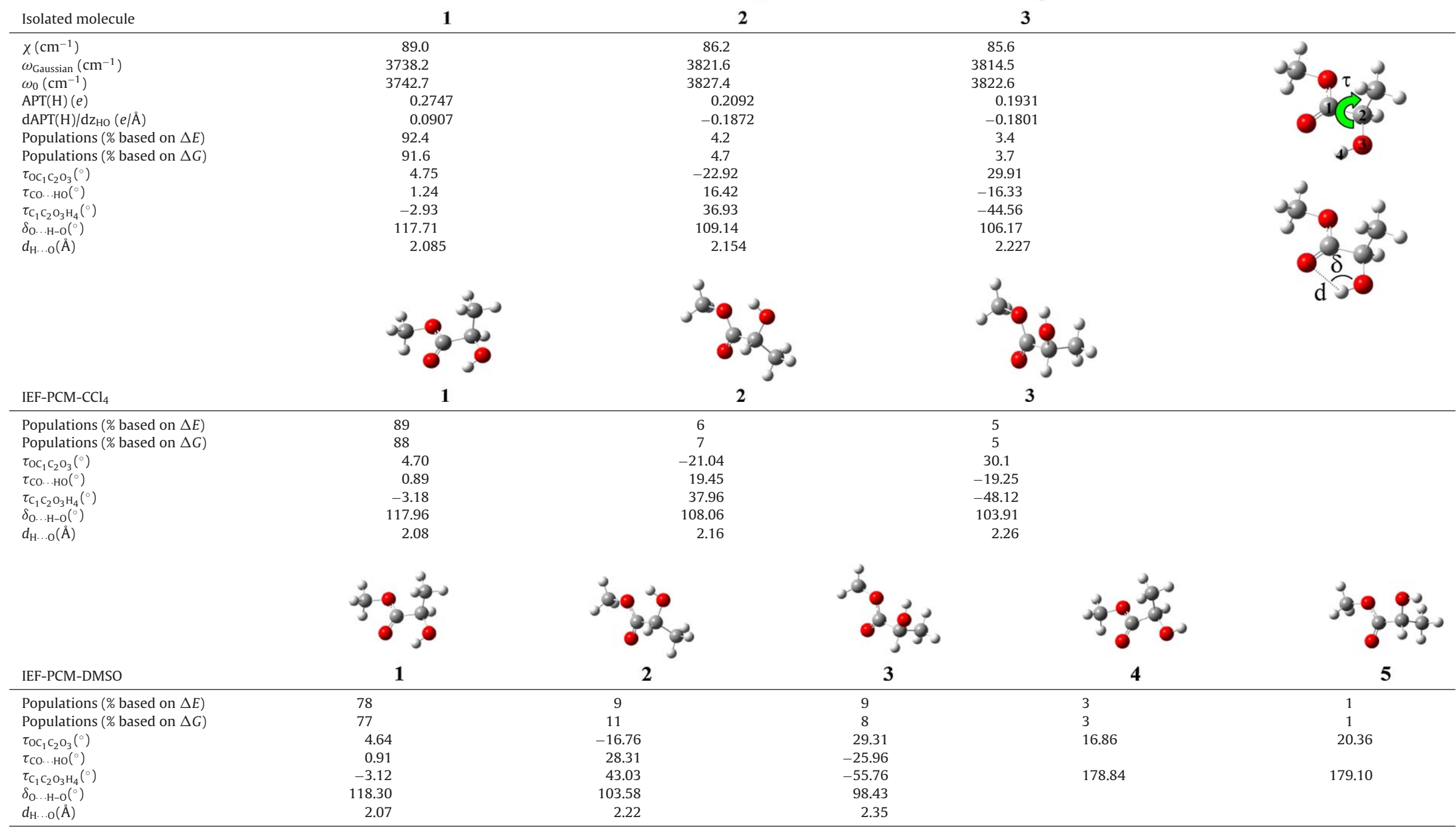



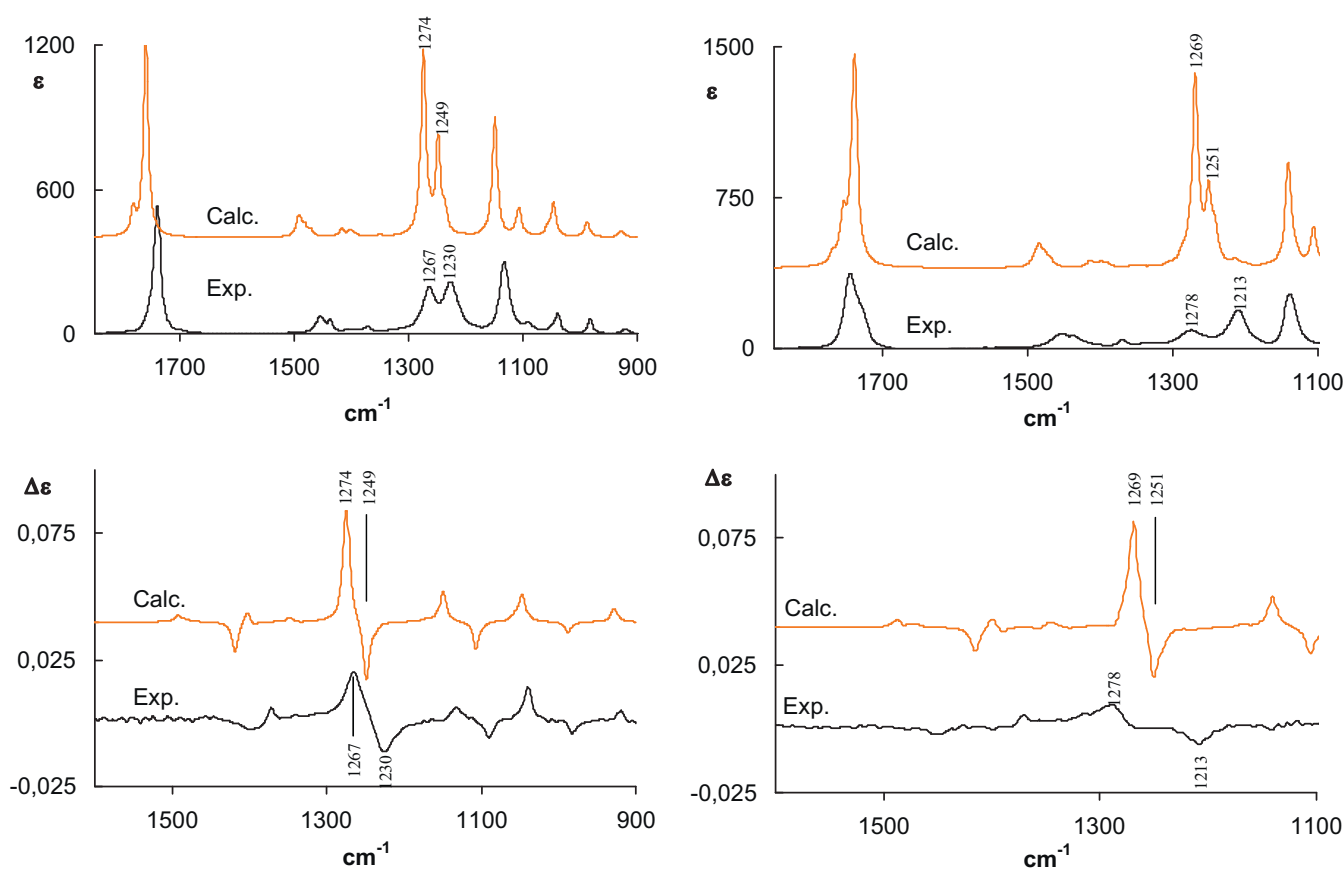

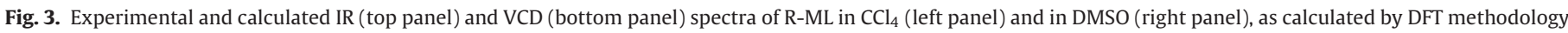
(for the modeling of the solvent we used the IEF-PCM method, see text). For the calculated spectra, only the weighted sum has been presented.

the frequency for the corresponding first overtone, we have from Ref. [20]:

$v_{1}=\omega_{0}-2 \chi$

$v_{2}=2 \omega_{0}-6 \chi$ for either $\alpha$ or $\beta$ conformer

We therefore conclude: $v_{2}=2 v_{1}-2 \chi$.
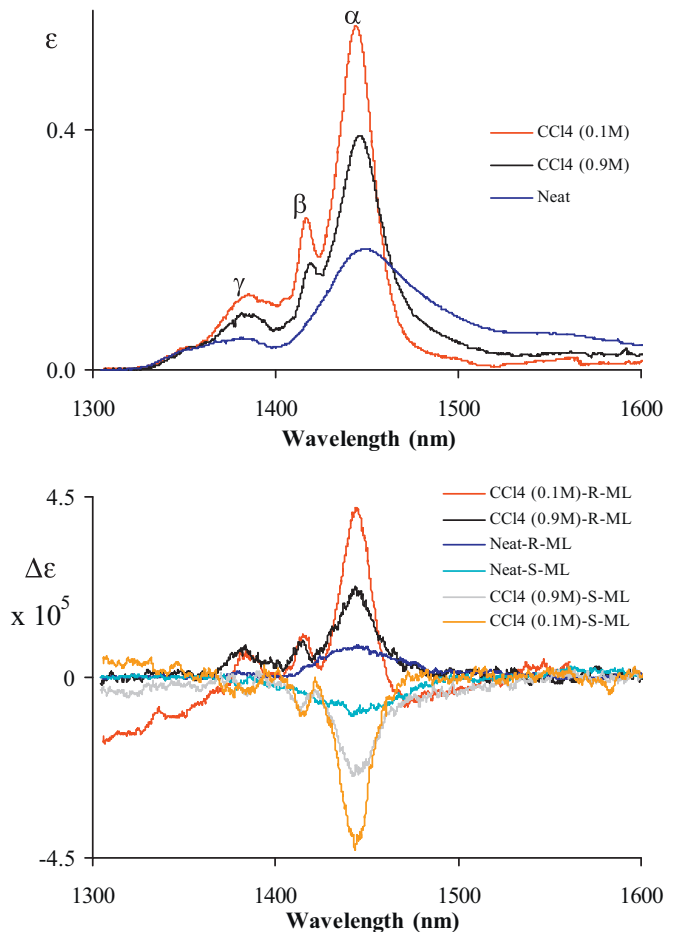

Indeed, assuming $\chi=88.6 \mathrm{~cm}^{-1}$, we obtain from the observed values of $v_{1}$ the results for $v_{2}$ reported in the fourth column, which compare well with the experimental values of $\nu_{2}$. Besides, since $v_{1 \alpha}+v_{1 \beta}=\omega_{0 \alpha}-2 \chi+\omega_{0 \beta}-2 \chi$, by just adding the observed values $v_{1 \alpha}$ and $v_{1 \beta}$, one obtains a frequency not too far from that of the band observed at ca. $1382 \mathrm{~nm}(1394 \mathrm{~nm})$, thus making the assignment to a combination state plausible. In order to make a less empirical analysis of the NIR data, we decided to undertake the
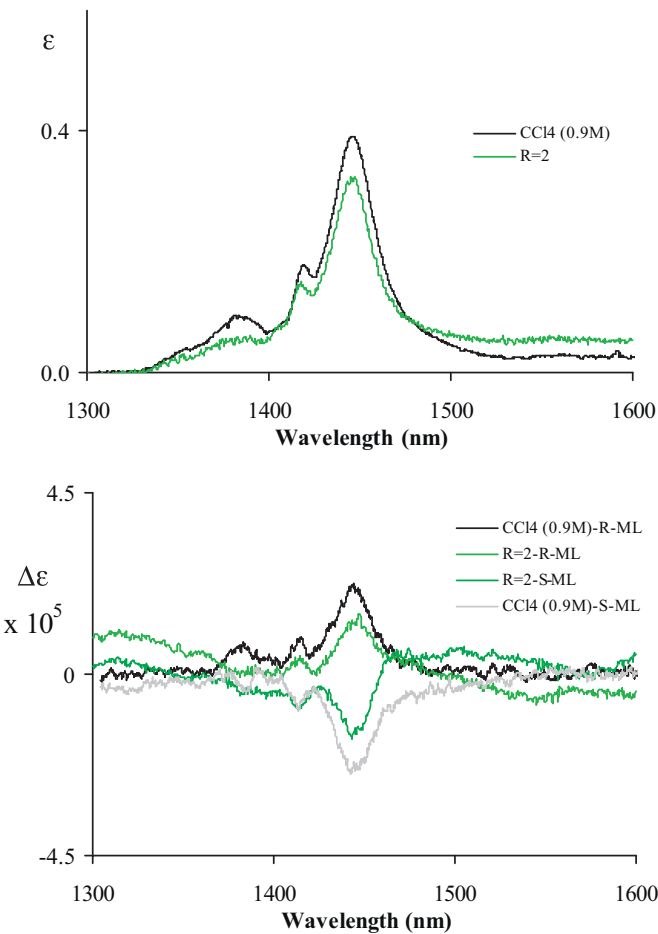

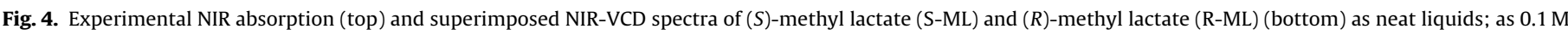
and $0.9 \mathrm{M} \mathrm{CCl}_{4}$ solutions and in $\mathbf{R}=2$ micellar molar ratio [ML]/[AOT] in the region $1300-1600 \mathrm{~nm}$. 

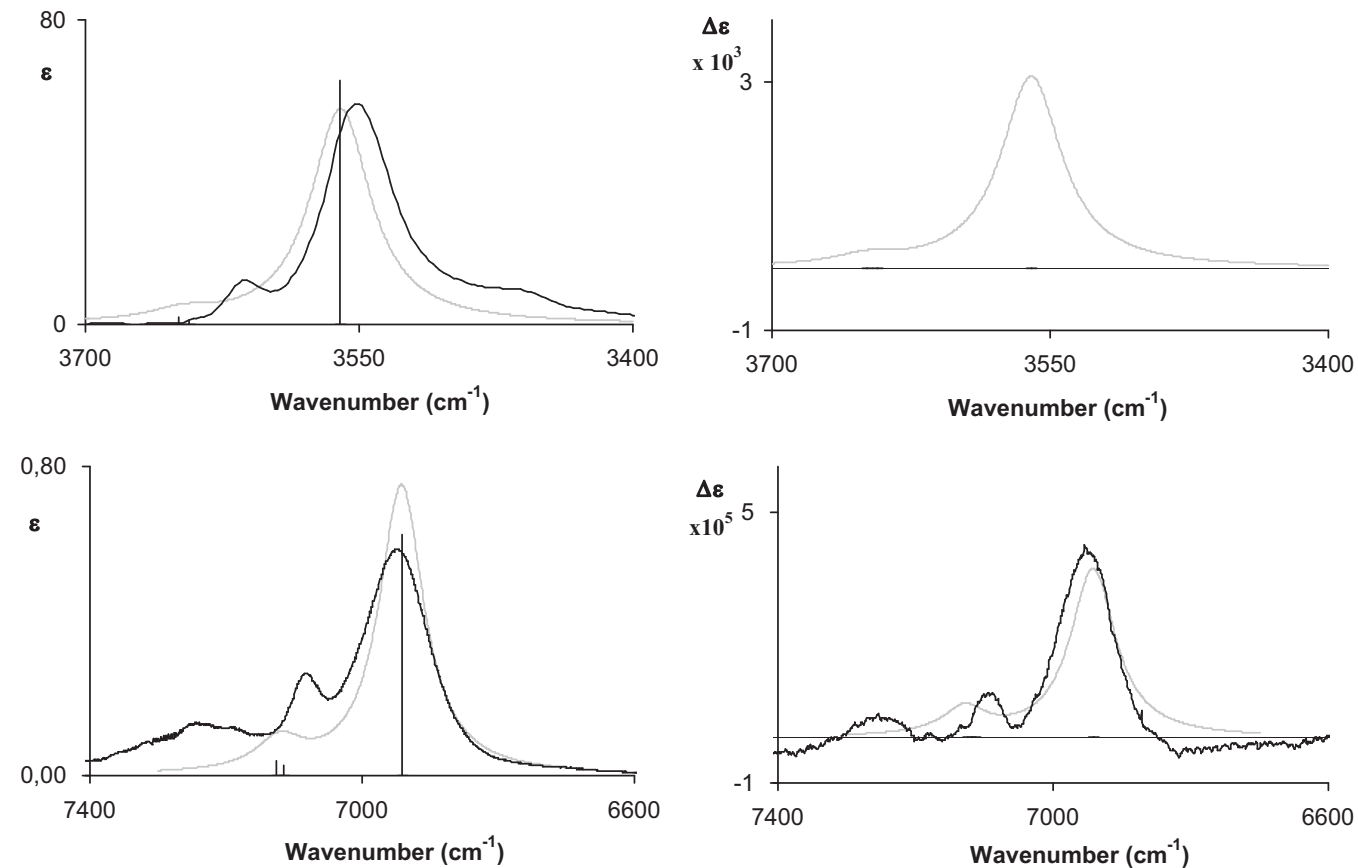

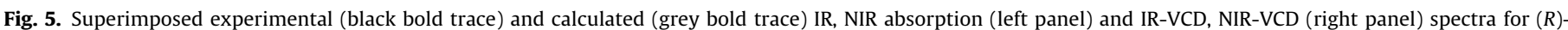

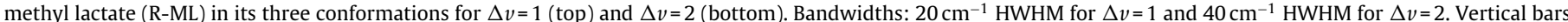

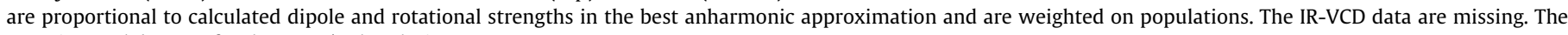
experimental data are for the $0.1 \mathrm{M} / \mathrm{CCl}_{4}$ solutions.

calculations of mechanical and electrical anharmonic parameters, as presented for the OH-stretching of borneol [15] in three conformational states, allowing us to arrive at a prediction of the IR, NIR and VCD spectra.

In Table 2 we report, for all three conformers, the calculated mechanical anharmonicity $\chi$, the harmonic frequency $\omega_{0}$ (either directly taken from the Gaussian 09 output or from our re-elaboration $[14,15])$ and two electrical harmonic and anharmonic parameters, namely $(\partial \mu / \partial q)$ (i.e. $\left.\operatorname{APT}_{z}(\mathrm{H})\right)$ and $\left(\partial^{2} \mu / \partial q^{2}\right)$ (i.e. $\left.\partial \mathrm{APT}_{z}(\mathrm{H}) / \partial q\right)\left(q=z_{\mathrm{OH}}\right)$. It is interesting to notice that for the first conformer, exhibiting internal hydrogen bonding $\mathrm{OH} \cdots \mathrm{O}=\mathrm{C}$ (Table 2 ), $\omega_{0}$ has the smallest value and the second derivative of $\mu$ is positive (the latter parameter is calculated to be negative for the other conformers and was calculated to be negative for all conformers of borneol, where no $\mathrm{H}$ bonding occurs [15]). From the behavior of APT and AAT components as function of the OH bond length $(z$ along $\mathrm{OH}, y$ in the $\mathrm{COH}$ plane, and $x$ perpendicular to the latter plane), we obtain: (1) that the $\mathrm{OH}$-bond electric dipole moment function for the conformer exhibiting $\mathrm{OH} \cdots \mathrm{O}=\mathrm{C}$ hydrogen bonding is different from the $\mathrm{OH}$-bond dipole moment function of the other two conformers (Table 2 and Fig. S2 of Supporting Information); the functions for the latter two conformers are more similar to the OH-dipole moment functions of borneol; (2) the behavior of the $x$-component of AAT is interesting: we notice that for conformer 1 such component is larger in absolute value, implying a large circulation of charge in the $(y, z)$ plane, which is close to the plane approximately defined by the set of $\mathrm{COH} \cdots \mathrm{O}=\mathrm{C}$ atoms. The use of these parameters allows us to produce the calculated spectra of Fig. 5, where we assign Lorentzian bands to the vibrational transitions in the fundamental $(\Delta v=1)$ and in the overtone $(\Delta v=2)$ regions for the three conformers, carrying a dipole or rotational strength equal to the area of the band, the latter being centered at the calculated frequencies and with bandwidths, empirically determined, of $20 \mathrm{~cm}^{-1}(\Delta v=1)$ and $40 \mathrm{~cm}^{-1}(\Delta v=2)$.

In that figure we report the weighted average (using the statistical weights in vacuo of Table 2) for the three conformers. Therein we compare them to the corresponding experimental IR and VCD spectra. As one may see, we obtain satisfactory results for the fully anharmonic calculations for the IR $(\Delta v=1)$ in absorption and for the NIR $(\Delta v=2)$, both in absorption and VCD. From these results, we obtain further and definite confidence in assigning the two IR, NIR and VCD peaks that we had called $\alpha$ and $\beta$ to the OH-stretching normal modes of the three conformers of the isolated molecule, one with strong internal $\mathrm{H}$-bonding. Having fully characterized the behavior of the $\mathrm{OH}$ stretching for the monomeric molecule, we conclude that this behavior is progressively decreasing in going to less and less dilute solutions and in presence of the surfactant, but is not completely lost. We observe that, as far as the NIR spectra are concerned, the data for the ternary systems $\mathrm{ML} / \mathrm{AOT} / \mathrm{CCl}_{4}$ are closest to the data for the $0.9 \mathrm{M}$ solutions both for the NIR and the NIR-VCD spectra apart from the band at about $1380 \mathrm{~nm}$ which is closer to the band observed in the pure liquid.

On the basis of the above results, we draw similar conclusions as obtained in first and second paragraphs of this section: some ML molecules interact with AOT molecules, some do not and, among the latter ones, some ML molecules interact with other ML molecules and some do not. We do not dare to be more quantitative, for example by using the intensity of the absorption or VCD band at $1442 \mathrm{~nm}$ in the different conditions, assuming that the $0.1 \mathrm{M} / \mathrm{CCl}_{4}$ solution represents of the isolated ML molecules. At this stage we can be content to conclude that isolated molecules are present both in solutions and in the ternary system.

\section{Conclusions}

In this work we have characterized $(S)$-methyl lactate and $(R)$ methyl lactate molecules in solution and have examined their interactions with AOT reverse micelles dispersed in $\mathrm{CCl}_{4}$. We have used three spectroscopic techniques, FT-IR absorption spectroscopy, VCD in the mid IR and VCD in the NIR. For the solution state we wish to point out that NIR-VCD spectroscopy and ab-initio calculations have allowed to observe different methyl lactate conformers, more clearly than done before in the case of borneol [15]. 
These conformers are observed to persist also in the tertiary system $\mathrm{ML} / \mathrm{AOT} / \mathrm{CCl}_{4}$.

Finally, all techniques employed here concur to monitor how ML molecules interact with AOT reverse micelles in limited number, the remaining ML molecules behaving either as isolated molecules or, to a larger extent, in association with other ML molecules.

\section{Acknowledgments}

We wish to thank Dr. Engineer Luca Berghella, University of Brescia, for significantly updating the electronics of the NIR apparatus. We would like to thank Italian "Ministero dell'Istruzione, dell'Università e della Ricerca” (MIUR) for financial support, under the auspices of the program PRIN 2008LYSEBR.

\section{Appendix A. Supplementary data}

Supplementary data associated with this article can be found, in the online version, at doi:10.1016/j.vibspec.2012.01.020.

\section{References}

[1] L.A. Nafie, T.B. Freedman, Vibrational optical activity: theory, in: N. Berova, K. Nakanishi, R.A. Woody (Eds.), Circular Dichroism. Principles and Applications, 2nd edition, Wiley-VCH, New York, 2000, pp. 97-132.

[2] T.B. Freedman, G.A. Balukjian, L.A. Nafie, J. Am. Chem. Soc. 107 (1985) 6213-6222.

[3] P.L. Polavarapu, C.S. Ewig, T. Chandramouly, J. Am. Chem. Soc. 109 (1987) $7382-7386$.

[4] Y. Nakao, H. Sugeta, Y. Kyogoku, Chem. Lett. 13 (1984) 623-626.

[5] R. Bursi, F.J. Devlin, P.J. Stephens, J. Am. Chem. Soc. 112 (1990) 9430-9432.
[6] M. Losada, Y. Xu, Phys. Chem. Chem. Phys. 9 (2007) 3127-3135.

[7] T.B. Adler, N. Bohro, M. Reiher, M.A. Suhm, Angew. Chem. Int. Ed. 45 (2006) 3440-3445.

[8] S. Abbate, G. Longhi, A. Ruggirello, V. Turco Liveri, Colloids Surf. A: Phys. Eng. Aspects 327 (2008) 44-50.

[9] S. Abbate, F. Castiglione, F. Lebon, G. Longhi, A. Longo, A. Mele, W. Panzeri, A Ruggirello, V. Turco Liveri, J. Phys. Chem. B 113 (2009) 3024-3033.

[10] P. Calandra, A. Longo, A. Ruggirello, V. Turco Liveri, J. Phys. Chem. B 108 (2004) 8260-8268.

[11] E. Castiglioni, F. Lebon, G. Longhi, S. Abbate, Enantiomer 7 (2002) 161-173.

[12] G. Longhi, R. Gangemi, F. Lebon, E. Castiglioni, S. Abbate, V.M. Pultz, D.A. Lightner, J. Phys. Chem. A 108 (2004) 5338-5352.

[13] M.J. Frisch, G.W. Trucks, H.B. Schlegel, G.E. Scuseria, M.A. Robb, J.R. Cheeseman, J.A. Montgomery Jr., T. Vreven, K.N. Kudin, J.C. Burant, J.M. Millam, S.S. Iyengar, J. Tomasi, V. Barone, B. Mennucci, M. Cossi, G. Scalmani, N. Rega, G.A. Petersson, H. Nakatsuji, M. Hada, M. Ehara, K. Toyota, R. Fukuda, J. Hasegawa, M. Ishida, T. Nakajima, Y. Honda, O. Kitao, H. Nakai, M. Klene, X. Li, J.E. Knox, H.P. Hratchian, J.B. Cross, V. Bakken, C. Adamo, J. Jaramillo, R. Gomperts, R.E. Stratmann, O. Yazyev, A.J. Austin, R. Cammi, C. Pomelli, J.W. Ochterski, P.Y. Ayala, K. Morokuma, G.A. Voth, P. Salvador, J.J. Dannenberg, V.G. Zakrzewski, S. Dapprich, A.D. Daniels, M.C. Strain, O. Farkas, D.K. Malick, A.D. Rabuck, K. Raghavachari, J.B. Foresman, J.V. Ortiz, Q. Cui, A.G. Baboul, S. Clifford, J. Cioslowski, B.B. Stefanov, G. Liu, A. Liashenko, P. Piskorz, I. Komaromi, R.L. Martin, D.J. Fox, T. Keith, M.A. AlLaham, C.Y. Peng, A. Nanayakkara, M. Challacombe, P.M.W. Gill, B. Johnson, W. Chen, M.W. Wong, C. Gonzalez, J.A. Pople, Gaussian09, A02 Version, Gaussian, Inc., Pittsburgh, PA, 2009.

[14] F. Gangemi, R. Gangemi, G. Longhi, S. Abbate, Vib. Spectrosc. 50 (2009) 257-267.

[15] F. Gangemi, R. Gangemi, G. Longhi, S. Abbate, Phys. Chem. Chem. Phys. 11 (2009) 2683-2689.

[16] D.M.P. Gigante, F. Long, L.A. Bodack, J.M. Evans, J. Kallmerten, L.A. Nafie, T.B. Freedman, J. Phys. Chem. A 103 (1999) 1523-1537.

[17] P. Calandra, E. Caponetti, D. Chillura Martino, P. D’Angelo, A. Minore, V. Turco Liveri, J. Mol. Struct. 522 (2000) 165-178.

[18] P. Zhang, P.L. Polavarapu, J. Phys. Chem. A 111 (2007) 858-871.

[19] C. Sandorfy, J. Mol. Struct. 614 (2002) 365-366.

[20] G. Herzberg, Molecular Spectra and Molecular Structure. I. Spectra of Diatomic Molecules, 2nd edition, Van Nostrand Reinhold Co., New York, 1950. 